\title{
Catheter-related Infection and Septicemia: Impact of Seasonality and Modifiable Practices from the DOPPS
}

\author{
Charmaine E. Lok, * Jyothi R. Thumma, $†$ Keith P. McCullough, † Brenda W. Gillespie, $\ddagger$ \\ Richard J. Fluck,§ Mark R. Marshall, П Hideki Kawanishi, ${ }^{\star *}$ Bruce M. Robinson,†‡ and \\ Ronald L. Pisoni† \\ *Toronto General Hospital and the University of Toronto, Toronto, Ontario, Canada, †Arbor Research \\ Collaborative for Health, Ann Arbor, Michigan, fUniversity of Michigan, Ann Arbor, Michigan, §Derby City \\ General Hospital, Derby, United Kingdom, IFaculty of Medical and Health Sciences, University of Auckland, \\ Auckland, New Zealand, and ${ }^{* *}$ Tsuchiya General Hospital, Hiroshima, Japan
}

\begin{abstract}
Hemodialysis (HD) catheter-related infection (CRI) and septicemia contribute to adverse outcomes. The impact of seasonality and prophylactic dialysis practices during high-risk periods remain unexplored. This multicenter study analyzed DOPPS data from 12,122 HD patients (from 442 facilities) to determine the association between seasonally related climatic variables and CRI and septicemia. Climatic variables were determined by linkage to National Climatic Data Center of National Oceanic and Atmospheric Administration data. Catheter care protocols were examined to determine if they could mitigate infection risk during high-risk seasons. Survival models were used to estimate the adjusted hazard ratio (AHR) of septicemia by season and by facility catheter dressing

protocol. The overall catheter-related septicemia rate was 0.47 per 1000 catheter days. It varied by season, with an AHR for summer of 1.46 (95\% CI: 1.19-1.80) compared with winter. Septicemia was associated with temperature $(\mathrm{AHR}=1.07 ; 95 \%$ CI: $1.02-1.13 ; p<0.001)$. Dressing protocols using chlorhexidine (AHR of septicemia $=0.55 ; 95 \%$ CI: $0.39-0.78)$ were associated with fewest episodes of CRI or septicemia. Higher catheterrelated septicemia in summer may be due to seasonal conditions (e.g., heat, perspiration) that facilitate bacterial growth and compromise protective measures. Extra vigilance and use of chlorhexidine-based dressing protocols may provide prophylaxis against CRI and septicemia.
\end{abstract}

Hemodialysis (HD) catheter placement rates and duration of use have increased over the last two decades $(1,2)$. Approximately $80 \%$ of North American patients initiate HD with a catheter, with recent data indicating catheter use rates of approximately $50 \%$, 4 months after dialysis initiation $(1,2)$. A potentially devastating complication of HD catheter use is infection. In HD patients, infection is the most common cause of morbidity and the second most common cause of death $(1,3)$. The risk of a sepsis-attributable death is 100 times that of the general population (4). HD catheters pose the highest risk of bacteremia, sepsis, and death compared with other vascular access types (5-10). It has been estimated that, annually,

Address correspondence to: Charmaine E. Lok, MD, Department of Medicine, Division of Nephrology, Toronto General Hospital, 8NU-844, 200 Elizabeth Street, Toronto, Ontario, M5G 2C4, Canada, Tel.: 416-340-4140, Fax: 416340-4999, or e-mail: charmaine.lok@uhn.ca.

Seminars in Dialysis-Vol 27, No 1 (January-February) 2014 pp. $72-77$

DOI: $10.1111 /$ sdi. 12141

(C) 2013 Wiley Periodicals, Inc. approximately $30 \%$ of patients using a central venous catheter experience a bacteremic or septic episode (9).

Despite proven prophylactic strategies (10) and widely distributed guidelines on the prevention of access-related infections (11-13), infection rates remain high and a significant concern. Identifying circumstances where patients are at high risk of catheter-related infection and their complications can help target prophylactic efforts. For example, infection-related hospitalization rates are particularly high in the first 2 months after dialysis initiation (14). Another predictable occurrence is the change in environmental heat and humidity associated with climate and the seasons. Climate and seasonal variability has long been associated with disease (15). Higher heat and humidity may be more conducive to bacterial growth and the risk of catheter-related septicemia and their complications. The impact of climate, seasonality, environmental temperature, and humidity on vascular access-related septicemia is unknown.

The Dialysis Outcomes and Practice Patterns Study (DOPPS) is a prospective, longitudinal, observational study of HD patients and facilities in 
12 countries. Furthermore, the DOPPS has a focus to determine readily modifiable means to improve patient outcomes. The global nature of the DOPPS is ideally poised to determine whether an association exists between catheter-related septicemia and seasonality and to determine modifiable practice patterns that may assist in reducing catheter-related septicemia in high-risk situations.

\section{Subjects and Methods}

Daily climate data from 1996 to 2002 were downloaded from the National Climatic Data Center (NCDC) of the National Oceanic and Atmospheric Administration (NOAA) website. At the time of dataset creation, this was the most current and available data; NCDC data were then linked to each DOPPS facility by mapping to the nearest weather station (16). The climate data set has daily climate data that include the mean, minimum, and maximum temperatures, absolute and relative humidity, dew point, precipitation, and station atmospheric pressure. Data from 1996 to 2002 were used to obtain average climate information for each day of the year for each DOPPS facility location. The data set also has data on the elevation from sea level, longitude, and latitude of each of the DOPPS facilities.

Data were abstracted from DOPPS phases I, II, and III (1996-2009). DOPPS is a prospective observational study in 12 countries (Australia, New Zealand, Belgium, Canada, France, Germany, Italy, Japan, Spain, Sweden, United Kingdom, and the United States), which is based on nationally representative samples of randomly selected dialysis facilities and patients. The DOPPS sampling plan and study design have been described previously (17).

To assess the association between catheter-related septicemia and seasonality and to determine modifiable practice patterns that may assist in reducing catheter-related septicemia, the current analyses were limited to tunneled catheter-related vascular access data of 12,122 HD patients from DOPPS I, II, and III. These patients had a total of 973 infections, 1682 septicemia hospitalizations, and 1862 vascular access-related infections other than septicemia. A final data set was created with one observation per person per week of tunneled catheter use (from tunneled catheter start date until catheter last use date or infection date in case of an infection event) with the average climate information for the facility location based on the day and month of the year. Seasons were defined using approximate dates for the solstices and equinoxes: Winter (December 22), Spring (March 22), Summer (June 22), and Fall (September 23). The differences in seasons were accounted for in Australia and New Zealand (i.e., data from winter in Australia were made to coincide with data from winter in North America, even though these seasons are 6 months apart). To look at vascular access-related practices and their associ- ation with catheter-related septicemia, we evaluated the dressing protocol/cleansing agent used by dialysis facilities for vascular access care. The five cleansing agents used by facilities included alcohol, betadyne, chlorhexidine, soap and water, and a combination of agents.

Catheter-related septicemia was defined as septicemia during or within 15 days after HD catheter use. Septicemia was defined by a hospitalization where at least one hospitalization diagnosis was "septicemia". As a sensitivity analysis, hospitalizations involving both septicemia and a list of other diagnoses indicating that the septicemia might be due to something other than the catheter (e.g., pneumonia, wound, abcess, cellulitis, etc.) were excluded. Catheter time at-risk and catheter-related septicemia were assigned to one of four seasons in each country.

\section{Statistical Methods}

Rates of catheter-related infection and septicemia per 1000 patient-days of follow-up with a catheter were plotted by calendar month. Both unadjusted and adjusted relative rates of catheter-related infection and septicemia by season were determined by using time-dependent Cox proportional hazards regression models. These models, updated weekly for climate and season data, were also used to determine the associations of facility daily climate data or facility vascular access cleansing solutions with catheter-related infection or septicemia. All models were adjusted for age, race, sex, 13 summary comorbidities (coronary artery disease, other cardiac disease, cerebrovascular disease, congestive heart failure, diabetes, gastrointestinal bleeding, hypertension, peripheral vascular disease, lung disease, neurologic disorder, cancer [excluding skin], psychiatric disease, recurrent cellulitis, and HIV infection), phase, and country. Robust variance estimates (sandwich estimator) were used to account for patient-level clustering of infection rates. Only the first infection or septicemia event per catheter was analyzed. All statistical analyses were performed using SAS software, version 9.2 (SAS Institute, Cary, NC).

\section{Results}

We analyzed 12,122 HD patients in 12 countries from DOPPS I, II, and III (1996-2009) and 2,070,254 catheter days at-risk. Overall, the catheter-related septicemia rate was $0.47 / 1000$ catheter days. The catheter-related septicemia rate was 0.52 / 1000 catheter days in North America, 0.35/1000 catheter days in Japan, and 0.41 per 1000 catheter days in Europe/Australia/New Zealand. The occurrence of catheter-related septicemia varied by month (Fig. 1) with an adjusted hazard ratio (AHR) for "summer" of 1.46 (95\% CI: 1.19-1.80) compared 


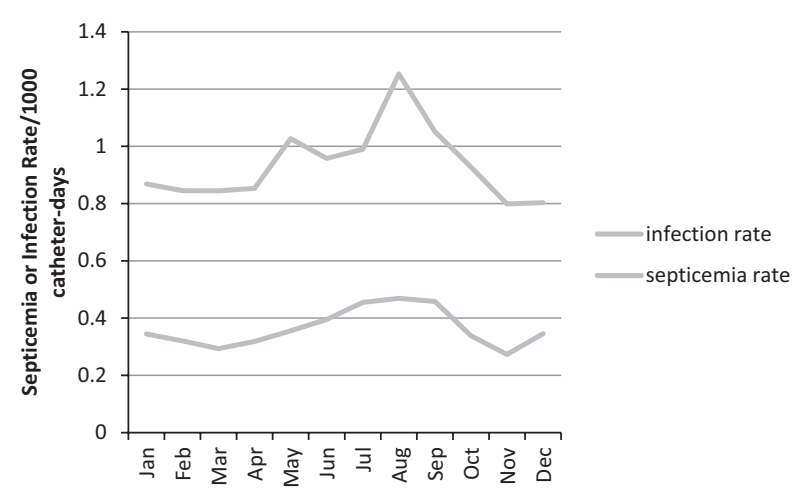

Fig. 1. Catheter-related infection and septicemia by month.

with "winter" (Table 1). As a sensitivity analysis, these results were redone excluding hospitalizations where the septicemia might have been due to another cause (e.g., pneumonia). The seasonal septicemia rates were $0.02-0.03 / 1000$ catheter days lower after excluding these admissions, but showed the same overall pattern. As a result of these rate changes, the AHR values after excluding these admissions increased by $\mathrm{AHR}=0-0.03$; for example, the AHR for "summer" compared with "winter" changed from 1.46 to 1.48 (95\% CI: $1.19-1.83$ ).

Climate-related variables had an association with catheter-related infections primarily through their association with temperature. Adjusting each variable for demographics and comorbid conditions, the following list of climate variables were significantly associated with catheter-related infections: absolute humidity (AHR 1.17 per $10 \mathrm{~g} / \mathrm{m}^{3}$ [95\% CI: 1.09 1.27; $p<0.001]$ ), latitude (AHR 0.86 per $10^{\circ}[95 \%$ CI: $0.79-0.95 ; p<0.001]$ ), mean temperature (AHR 1.08 per $10^{\circ}$ [95\% CI: $\left.1.04-1.12 ; p<0.001\right]$ ), minimum temperature (AHR 1.08 per $10^{\circ}[95 \% \mathrm{CI}$ : $1.05-1.12 ; p<0.001]$ ), maximum temperature (AHR 1.07 per $10^{\circ}$ [95\% CI: $\left.1.04-1.12 ; p<0.001\right]$ ), and mean dew point (AHR 1.07 per $10^{\circ} \mathrm{F}[95 \%$ CI: $1.04-1.11 ; p<0.001])$. When minimum temperature was included-along with the demographics and comorbid factors - as a covariate in the models using the various climate factors (Table 2), none of the other factors were statistically significant, indicating that humidity and dew point were only associated with infections through their correlation with temperature. These results were similar to those obtained when septicemia alone, instead of any infection, was used as the outcome. There appeared to be a statistically significant relationship between latitude and septicemia $(p=0.03)$, but not infection $(\mathrm{p}=0.39)$.

Catheter-related septicemia was lower with use of chlorhexidine (AHR $=0.55 ; 95 \%$ CI: 0.39-0.78) or iodine-based antiseptics, e.g., betadyne (AHR = 0.57; 95\% CI: $0.42,0.78)$, versus alcohol solutions, with similar AHR for combined cleansing agents $($ AHR $=0.60 ; 95 \%$ CI: 0.36, 0.98). Soap and water was only used in six of the facilities, which may be why their results $(\mathrm{AHR}=1.37 ; 95 \%$ CI: 0.51-3.7) were not significantly worse than alcohol. Betadyne and alcohol were used in 68 of the facilities; this combination trended toward lower catheter-related septicemia when compared with alcohol alone (AHR $=0.72,95 \%$ CI: 0.51-1.02). The combination was also associated with significantly higher catheter-related septicemia rates than betadyne alone $(\mathrm{AHR}=1.26,95 \% \mathrm{CI}: 1.00-1.58)$. There was no interaction with any of the cleansing agents and the seasons.

\section{Discussion}

This study found distinct seasonal variation in the incidence of catheter-related septicemia, with an increased risk of $46 \%$ during the summer months. This association persisted around the globe, even after accounting for differences in seasons based on geographic region (e.g., where July and August is summer in the Northern hemisphere, but winter in the Southern one). Consistent with the seasonal findings was the positive association between temperature and catheter-related infections. Furthermore, the study identified certain facility practice patterns that reduced the risk of infection, such as catheter care with appropriate antisepsis.

While the general relationship between season and disease has been long recognized (15), seasonality has been only provisionally reported in relation to access-related infection in the HD population $(18,19)$. However, in the peritoneal dialysis literature, peritonitis rates have been found to vary with seasons. Both Kim et al. and Szeto et al. described increased incidence during seasons of high temperature and humidity $(20,21)$. Such findings are consistent with our finding of higher access-related infections and septicemia rates during the summer. Furthermore, the organisms that demonstrate seasonality for peritonitis are also those that cause catheter-related septicemia. In the peritoneal dialysis

TABLE 1. Rate of first recorded catheter-related infection and septicemia (per 1000 catheter days) by season

\begin{tabular}{lllllr}
\hline Season & \multicolumn{1}{c}{ Fall } & Winter & Spring & Summer & Total \\
\hline Infection rate & 0.82 & 0.83 & 0.90 & 1.07 & 0.91 \\
Septicemia rate & 0.32 & 0.31 & 0.34 & 0.44 & 0.35 \\
Infection AHR & $1.00(0.87-1.14)$ & 1 (ref) & $1.10(0.97-1.26)$ & $1.32(1.16-1.50)$ & $1.46(1.19-1.80)$ \\
Septicemia AHR & $1.08(0.86-1.34)$ & $1(\mathrm{ref})$ & $1.13(0.91-1.41)$ & & \\
\hline
\end{tabular}

$\mathrm{AHR}=$ Adjusted hazard ratio for each season calculated using Cox time-dependent models on infection and septicemia, controlling for DOPPS phase, country, age, sex, race (Black versus non-Black), and 13 comorbid conditions. 
TABLE 2. Relationship between climate measures and CVC-related infections or septicemia, adjusting for minimum temperature

\begin{tabular}{|c|c|c|c|c|c|c|}
\hline & \multicolumn{3}{|c|}{ Infection } & \multicolumn{3}{|c|}{ Septicemia } \\
\hline & $\mathrm{AHR}^{\mathrm{a}}$ & $95 \% \mathrm{CI}$ & $p$-value & $\mathrm{AHR}^{\mathrm{a}}$ & $95 \% \mathrm{CI}$ & $p$-value \\
\hline Absolute humidity (per $10 \mathrm{~g} / \mathrm{m}^{3}$ ) & 0.95 & $(0.77,1.18)$ & 0.66 & 0.86 & $(0.61,1.2)$ & 0.37 \\
\hline Relative humidity (per $10 \%$ ) & 0.98 & $(0.93,1.02)$ & 0.33 & 0.98 & $(0.9,1.06)$ & 0.63 \\
\hline Latitude (per $10^{\circ}$ ) & 0.95 & $(0.86,1.06)$ & 0.39 & 1.20 & $(1.02,1.41)$ & 0.03 \\
\hline Mean temp (per $\left.10^{\circ} \mathrm{F}\right)$ & 1.00 & $(0.84,1.17)$ & 0.96 & 1.08 & $(0.83,1.41)$ & 0.56 \\
\hline Max. temp (per $\left.10^{\circ} \mathrm{F}\right)$ & 0.98 & $(0.89,1.07)$ & 0.62 & 1.03 & $(0.9,1.19)$ & 0.66 \\
\hline Min. temp (per $\left.10^{\circ} \mathrm{F}\right)$ & 1.08 & $(1.05,1.12)$ & $<.001$ & 1.07 & $(1.02,1.13)$ & 0.01 \\
\hline Difference in temp (per $\left.10^{\circ} \mathrm{F}\right)$ & 0.98 & $(0.89,1.07)$ & 0.62 & 1.03 & $(0.9,1.19)$ & 0.66 \\
\hline Elevation (per $100 \mathrm{~m}$ ) & 1.00 & $(0.98,1.02)$ & 1.00 & 1.00 & $(0.97,1.03)$ & 0.95 \\
\hline Mean dew point (per $\left.10^{\circ} \mathrm{F}\right)$ & 0.92 & $(0.81,1.05)$ & 0.23 & 0.95 & $(0.77,1.18)$ & 0.65 \\
\hline Total precipitation (per inch) & 1.17 & $(0.86,1.6)$ & 0.32 & 1.52 & $(0.96,2.4)$ & 0.07 \\
\hline
\end{tabular}

${ }^{\mathrm{a} C}$ Cox time-dependent models on infection and septicemia control for DOPPS phase, country, age, male, race (Black versus non-Black), minimum temperature, and 13 comorbid conditions.

literature, S. epidermidis, gram-negative organisms, and culture-negative infections were most frequent during hot months of the year, while $S$. aureus was uniformly distributed throughout the year (22). Staphylococcus aureus is the culprit organism identified in approximately one-third (27-39\%) of bacteremias in dialysis patients $(23,24)$. Our study was unable to examine the type of organism causing episodes of septicemia. However, DOPPS data are derived from a random sampling of dialysis patients and, as such, are representative of the general facility dialysis population from a given region, and probably, the organisms contributing to septicemia.

It is likely that a hot climate associates the summer months with a high incidence of catheterrelated infections in both peritoneal and HD patients $(21,25)$. Sweat and dirt are more likely to accumulate around the catheter exit site during the summer months $(20,21,26)$. Protective barriers, such as bandages, gauzes and/or antibiotic ointments, are less likely to remain intact on dialysis patients as they sweat. Further potential infection risks occur when patients attempt to replace lost dressings under suboptimal conditions. Heat and humidity may enhance growth of bacteria and fomites on catheter tubing, hubs, caps, and other reservoirs, and increase the risk of exit site, peritoneal, or HD catheter-related infections. This clinical postulate is consistent with the pathophysiology of catheterrelated infections and sepsis, with biofilm as a central requirement $(10,27)$.

A biofilm is an organized assembly of surfaceassociated microbial cells (micro-colonies) enclosed in a protective extracellular polysaccharide matrix. They adhere to and develop on living and nonliving materials in contact with liquid or damp environments. They are found in natural ecologic, manmade industrial and human environments. Biofilms are involved in over $65 \%$ of infections in the body (28) and have been long established to play a pathologic role in device-associated infections (29). Heat and atmospheric conditions are known to affect biofilm formation (30). Increasing temperature can accelerate colonization and increase the quantity and thickness of the biofilm biomass (31-35). Fur- thermore, the colony count of skin bacteria (e.g., S. epidermidis, S. aureus) at the internal jugular and subclavian exit site has been documented to be approximately $1000-10,000 \mathrm{cfu} / \mathrm{cm}^{2}$ (36), with $80 \%$ residing in the top five layers of the stratum corneum (37). The deeper hair follicles and sebaceous glands may be stimulated in heated conditions and harbor the remaining $20 \%$ of organisms within biofilms that provide extra protection against antiseptic agents (38-40). HD patients using a catheter during the summer months with high temperatures and humidity are therefore in the ideal setting for biofilm growth and dispersion, culminating ultimately in a higher rate of infection and septicemia.

While the climate and seasons cannot be altered, the associated environmental factors, particularly in relation to the pathophysiology of infection and septicemia in HD patients using a catheter, should be considered in prophylactic strategies. Patients at high risk, such as those with a prior bacteremic episodes $(7,41)$ recent hospitalizations (42), immunocompromised state $(41,42)$, and poor hygiene $(7,42)$, should have their catheter care and evaluation performed by qualified dialysis staff. Excellent catheter care and appropriate antisepsis can reduce microorganism entry, adherence to the catheter, and biofilm development via extraluminal routes available by intercutaneous breaks at the catheter exit site. This is supported by the findings in our study, where the use of chlorhexidine and iodine-based cleansing agents, compared with alcohol-based cleansing solutions, protected against catheterrelated infections. Our findings are consistent with randomized studies and meta-analysis of prophylactic cleansing agents used in high-risk settings, including in the HD population (43-45). Of note, our study extends the findings of the early landmark randomized controlled trial of povidoneiodine that demonstrated a reduction in exit-site infections, catheter tip colonization and bacteremias in a single center using temporary catheters (45).Common sense prevention such as hand washing prior to replacing dressings easily dislodged by excessive sweat, meticulous catheter care, use of air conditioning and dehumidifiers, and replacing damp 
clothing or carefully toweling dry sweaty necks and chests can be easily taught and implemented to patients and dialysis healthcare providers. Proven strategies for access-related infection prophylaxis have been well-documented $(10,27)$.

\section{Limitations}

This was an observational study that linked DOPPS dialysis units to the closest weather station. The precise temperature and humidity on the day of diagnosed septicemia may not have been possible. Only one observation per person per week of catheter use was analyzed, and the climate data were averaged, based on the location and time of the year. Therefore, the relationships between infection rates and climate variables are likely to have been attenuated by statistical noise, which would tend to increase the chances of obtaining a nonsignificant result. There may have been changes in practice pattern over time that is not captured by DOPPS data, such as use of hand sanitizers or treatment for nasal $S$. aureus carriers. However, such changes would not specifically and consistently affect catheterrelated infections only in a particular season over several years. However, the observational nature of this study limits our ability to comprehensively adjust for important factors, such as personal hygiene or socioeconomic status, which may impact infection rates. In addition, analyses of recurrent infections within the same person were limited as these infections are plausibly not independent of each other, even though only the first infection attributed to a single catheter was included. Recurrent or new infections in the same catheter would have been excluded. Thus, given that having had a catheter-related infection increases the risk of a subsequent infection, the overall rate of catheter-related infection or septicemia attributed to a catheter may be underestimated. Analyses of time-to-first infection rates within a season are also limited, in that multiple infections for a particular patient within a season would not have been counted. Thus, the true rate of catheter-related infections may be underestimated. Lastly, it may be possible that some catheter-related infections were really septicemia managed at an outpatient facility rather than being hospitalized. However, catheter-related infections and septicemia had consistently similar seasonal trends.

\section{Conclusions}

The higher rate of catheter-related septicemia in summer may be due to higher heat and perspiration, potentially facilitating bacterial growth and compromising protective measures. Extra care and vigilance by staff may reduce catheter-related sepsis during this high-risk season. Prophylaxis should include iodine-based antiseptic agents or chlorhexidine cleansing of the catheter and exit site, applied by suitably trained health care workers.

\section{Acknowledgments}

Heather Van Doren, MFA, a senior medical editor with Arbor Research Collaborative for Health, provided editorial assistance on this manuscript.

\section{Declaration of Presentation}

The results presented in this paper have not been published previously in whole or part, except in abstract format (46).

\section{Funding}

The DOPPS is administered by Arbor Research Collaborative for Health and is supported by Scientific Research Grants from Amgen (since 1996), Kyowa Hakko Kirin (since 1999, in Japan), Sanofi/Genzyme (since 2009), Abbott (since 2009), Baxter (since 2011), and Vifor Fresenius Renal Pharma (since 2011), without restrictions on publications.

\section{Financial Disclosure}

Bruce Robinson has received a speaker fee from Kyowa Hakko Kirin. All the remaining authors declare no competing interests.

\section{References}

1. USRDS: U.S. Renal Data System: USRDS 2012 Annual Data Report: Atlas of Chronic Kidney Disease and End-Stage Renal Disease in the United States, National Institutes of Health, vol. 2. Bethesda, MD: National Institute of Diabetes and Digestive and Kidney Diseases, 2012

2. CORR: Canadian Organ Replacement Register, 2010 Report. Ottawa, Ontario: Canadian Institute for Health Information, 2010

3. Schaubel DE, Fenton SSA: Trends in mortality rates on hemodialysis in Canada, 1981-1997. Kidney Int 57:S66-S73, 2000

4. Sarnak MJ, Jaber BL: Mortality caused by sepsis in patients with endstage renal disease compared with the general population. Kidney Int 58:1758-1764, 2000

5. Tokars JI, Miller ER, Stein G: New national surveillance system for hemodialysis-associated infections: initial results. Am J Infect Control 30:288-295, 2002

6. Dopirak M, Hill C, Oleksiw M, Dumigan D, Arvai J, English E, Carusillo E, Malo-Schlegel S, Richo J, Traficanti K, Welch B, Cooper B: Surveillance of hemodialysis-associated primary bloodstream infections: the experience of ten hospital-based centers. Infect Control Hosp Epidemiol 23:721-724, 2002

7. Taylor G, Gravel D, Johnston L, Embil J, Holton D, Paton S, Canadian Nosocomial Infection Surveillance P, Canadian Hospital Epidemiology C: Incidence of bloodstream infection in multicenter inception cohorts of hemodialysis patients. Am $J$ Infect Control 32:155-160, 2004

8. Dhingra RK, Young EW, Hulbert-Shearon TE, Leavey SF, Port FK: Type of vascular access and mortality in U.S. hemodialysis patients. Kidney Int 60:1443-1451, 2001

9. Ishani A, Collins AJ, Herzog CA, Foley RN: Septicemia, access and cardiovascular disease in dialysis patients: the USRDS Wave 2 study. Kidney Int 68:311-318, 2005

10. Lok CE, Mokrzycki MH: Prevention and management of catheterrelated infection in hemodialysis patients. Kidney Int 79:587-598, 2011 
11. Allon M: Treatment guidelines for dialysis catheter-related bacteremia: an update. Am J Kidney Dis 54:13-17, 2009

12. Mermel LA, Allon M, Bouza E, Craven DE, Flynn P, O'Grady NP, Raad II, Rijnders BJ, Sherertz RJ, Warren DK: Clinical practice guidelines for the diagnosis and management of intravascular catheterrelated infection: 2009 Update by the Infectious Diseases Society of America. Clin Infect Dis 49:1-45, 2009

13. Division of Nosocomial and Occupational Infectious Diseases, Bureau of Infectious Diseases, Laboratory Centre for Disease Control, Health Canada: Preventing infections associated with indwelling intravascular access devices. Can Commun Dis Rep 23(Suppl 8): i-iii, 1-32, i-iv, 116, 1997

14. USRDS: U.S. Renal Data System: USRDS 2008 Annual Data Report: Atlas of Chronic Kidney Disease and End-Stage Renal Disease in the United States, National Institutes of Health. Bethesda, MD: National Institute of Diabetes and Digestive and Kidney Diseases, 2008

15. Stallybrass CO: Season and disease. Proc $R$ Soc Med 21:1185-1210, 1928

16. NOAA No. http://www.ncdc.noaa.gov/data-access. Accessed September 12, 2008.

17. Young EW, Goodkin DA, Mapes DL, Port FK, Keen ML, Chen K, Maroni BL, Wolfe RA, Held PJ: The Dialysis Outcomes and Practice Patterns Study (DOPPS): an international hemodialysis study. Kidney Int 57:S74-S81, 2000

18. Agarwal AK BW, Cosio FG, Van Cleef SS, Hebert LA: Seasonal variation in incidence of infection in long term tunneled catheters and gore-tex grafts used as hemodialysis vascular access. J Am Soc Nephrol 10: 200A, 1999

19. Lawrence C, Nipah RG, Farrell M, Almond MK: Seasonal variations in the rate of dialysis catheter related bacteraemia: The influence of ambient temperature. J Am Soc Nephrol 18, 2007

20. Kim MJ, Song JH, Park YJ, Kim GA, Lee SW: The influence of seasonal factors on the incidence of peritonitis in continuous ambulatory peritoneal dialysis in the temperate zone. Adv Perit Dial 16:243-247, 2000

21. Szeto CC, Chow KM, Wong TY, Leung CB, Li PK: Influence of climate on the incidence of peritoneal dialysis-related peritonitis. Peritoneal dial Int 23:580-586, 2003

22. Chan MK, Chan CY, Cheng IK, Ng WS: Climatic factors and peritonitis in CAPD patients. Int J Artif Organs 12:366-368, 1989

23. Hoen B, Paul-Dauphin A, Hestin D, Kessler M: EPIBACDIAL: a multicenter prospective study of risk factors for bacteremia in chronic hemodialysis patients. J Am Soc Nephrol 9:869-876, 1998

24. Danese MD, Griffiths RI, Dylan M, Yu HT, Dubois R, Nissenson AR: Mortality differences among organisms causing septicemia in hemodialysis patients. Hemodial Int 10:56-62, 2006

25. Alves FR, Dantas RC, Lugon JR: Higher incidence of catheter-related infections in a tropical climate. Adv Perit Dial 9:244-247, 1993

26. Piraino B, Bernardini J, Sorkin M: A five-year study of the microbiologic results of exit site infections and peritonitis in continuous ambulatory peritoneal dialysis. Am J Kidney Dis 10:281-286, 1987

27. Pronovost $P$, Needham D, Berenholtz S, Sinopoli D, Chu H, Cosgrove S, Sexton B, Hyzy R, Welsh R, Roth G, Bander J, Kepros J, Goeschel C: An intervention to decrease catheter-related bloodstream infections in the ICU. N Engl J Med 355:2725-2732, 2006

28. Costerton JW, Stewart PS, Greenberg EP: Bacterial biofilms: a common cause of persistent infections. Science 284:1318-1322, 1999
29. Donlan RM: Biofilms and device-associated infections. Emerg Infect Dis 7:277-281, 2001

30. Kaali P, Stromberg E, Sigbritt K: Prevention of biofilm associated infections and degradation of polymeric materials used in biomedical application. In: Laskovski AN (ed). Biomedical Engineering, Trends in Materials Science. Sweden: InTech, 2011:1-28

31. Diaz Villanueva V, Font J, Schwartz T, Romani AM: Biofilm formation at warming temperature: acceleration of microbial colonization and microbial interactive effects. Biofouling 27: 59-71, 2011

32. Han TH, Lee JH, Cho MH, Wood TK, Lee J: Environmental factors affecting indole production in Escherichia coli. Res Microbiol 162:108116,2011

33. Norf $\mathrm{H}$, Arndt $\mathrm{H}$, Weitere M: Impact of local temperature increase on the early development of biofilm-associated ciliate communities. Oecologia 151:341-350, 2007

34. Aguilera A, Zettler E, Gomez F, Amaral-Zettler L, Rodriguez N, Amils R: Distribution and seasonal variability in the benthic eukaryotic community of Rio Tinto (SW, Spain), an acidic, high metal extreme environment. Systematic Appl Microbiol 30:531-546, 2007

35. Ratkowsky DA, Olley J, McMeekin TA, Ball A: Relationship between temperature and growth rate of bacterial cultures. J Bacteriol 149:1-5, 1982

36. Maki DG, Ringer M: Evaluation of dressing regimens for prevention of infection with peripheral intravenous catheters. Gauze, a transparent polyurethane dressing, and an iodophor-transparent dressing. JAMA 258:2396-2403, 1987

37. Brown E, Wenzel RP, Hendley JO: Exploration of the microbial anatomy of normal human skin by using plasmid profiles of coagulasenegative staphylococci: search for the reservoir of resident skin flora. J Infect Dis 160:644-650, 1989

38. Hall-Stoodley L, Stoodley P: Biofilm formation and dispersal and the transmission of human pathogens. Trends Microbiol 13:7-10, 2005

39. Messager S, Hann AC, Goddard PA, Dettmar PW, Maillard JY: Use of the 'ex vivo' test to study long-term bacterial survival on human skin and their sensitivity to antisepsis. J Appl Microbiol 97:1149-1160, 2004

40. Hendley JO, Ashe KM: Effect of topical antimicrobial treatment on aerobic bacteria in the stratum corneum of human skin. Antimicrob Agents Chemother 35:627-631, 1991

41. Marr KA SD, Conlon PJ, Corey GR, Schwab SJ, Kirkland KB: Catheter-related bacteremia and outcome of attempted catheter salvage in patients undergoing hemodialysis. Ann Intern Med 127:275-280, 1997

42. Tokars JI, Light P, Anderson J, Miller ER, Parrish J, Armistead N, Jarvis WR, Gehr T: A prospective study of vascular access infections at seven outpatient hemodialysis centers. Am J Kidney Dis 37:12321240, 2001

43. Darouiche RO, Wall MJ Jr, Itani KM, Otterson MF, Webb AL, Carrick MM, Miller HJ, Awad SS, Crosby CT, Mosier MC, Alsharif A, Berger DH: Chlorhexidine-Alcohol versus Povidone-Iodine for Surgical-Site Antisepsis. N Engl J Med 362:18-26, 2010

44. Chaiyakunapruk N, Veenstra DL, Lipsky BA, Saint S: Chlorhexidine compared with povidone-iodine solution for vascular catheter-site care: a meta-analysis. Ann Intern Med 136:792-801, 2002

45. Levin A, Mason AJ, Jindal KK, Fong IW, Goldstein MB: Prevention of hemodialysis subclavian vein catheter infections by topical povidone-iodine. Kidney Int 40:934-938, 1991

46. Stanley K: Design of randomized controlled trials. Circulation 115:1164-1169, 2007 